Flocking of lifeless matter

Nature Commun. 5, 4688 (2014)

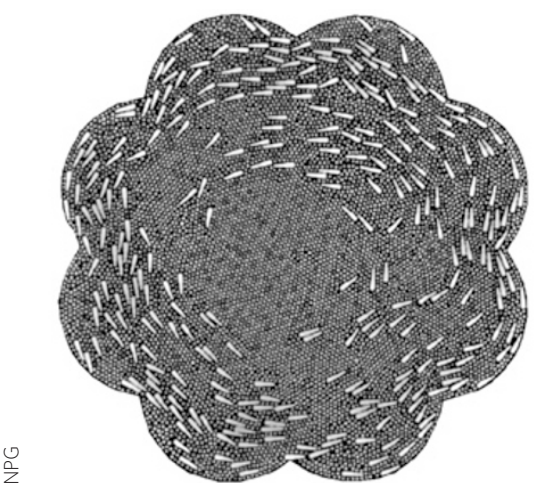

Analogies between birds moving in the same direction and aligned phases in condensed matter (such as iron's ferromagnetic phase, where all spins are on average aligned) have been made for about two decades. Now, Nitin Kumar et al. show another example of collective self-organization in inanimate matter: the spontaneous alignment of macroscopic tapered metal rods lying on a mechanically vibrated bed of smaller metal beads. The researchers find that on increasing the surface coverage of either the rods or the underlying beads, the system undergoes a 'flocking' transition from a disordered to an aligned configuration (even at low concentration of rods). They also show with computer simulation and hydrodynamic theory that the rods transmit information about their orientation by dragging nearby beads, whose flow causes the reorientation of neighbouring rods. Such 'hydrodynamic coupling' has been shown to lead to oriented motion in self-propelled rolling colloidal particles, and has analogies to the coupling of local velocity fluctuations in neighbouring birds within a flock.

\section{All-optical dynamic imaging Nature Photon. 8, 695-700 (2014)}

Time-resolved measurements of ultrafast events can be obtained by using two different paths: either by recording a short burst of images at a high frame rate by using burstmode cameras or by producing a timeresolved movie from repetitive pump-probe measurements, by varying the pump-probe time delay. Either way, the time-resolved imaging of events in the pico- and femtosecond regimes has so far been prevented by the limit on the maximum speed of the mechanical and electrical components of high-speed cameras. Now, Keisuke Goda, Ichiro Sakuma and colleagues propose an all-optical burstmode camera that avoids the use of mechanical and electrical components. In their approach, a femtosecond laser pulse is discretized in sub-pulses that are used to image the sample at successive times within the femtosecond laser pulse. These discretized pulses are then spatially separated and analysed by an image sensor. In this way, plasma dynamics on the picosecond timescale and lattice vibrations on the femtosecond timescale were directly imaged. One can also imagine a number of future applications of this technique in ultrafast and non-repeatable processes.

\section{Contacts matter}

\section{ACS Nano 8, 8653-8661 (2014)}

Because of their good optoelectronic properties, semiconducting monolayers of transition metal dichalcogenides are being extensively used for the realization of efficient phototransistors. However, the lightconversion performance of these devices is affected by the metals used to contact the semiconductors. Now, Wenjing Zhang and collaborators have studied the potential energy

\title{
Breaking barriers
}

Angew. Chem. Int. Ed. http://doi.org/f2ts2f (2014)

Marine mussels stick to underwater surfaces and hence must have mechanisms by which they can overcome the repulsive forces usually prevalent between adhesive materials and hydrated surfaces. Mimicking this 'wet adhesion' with synthetic adhesives remains challenging, however, because methods to analyse the interaction between mussel proteins and the hydrated layers of solid surfaces by, for example, atom force microscopy or surface force apparatus experiments, apply disruptive forces to the repulsive layers and mask the mechanisms involved. Now, Songi Han and colleagues use force-free conditions to analyse the diffusion dynamics of surface water between various mussel foot proteins and polystyrene surfaces that are suspended in bulk water. In the approach, which combines Overhauser dynamic nuclear polarization and electron spin resonance spectroscopy, the interfacial interactions are observed. The researchers find that the most hydrophobic protein, which has a weaker hydrated layer surrounding it than the hydrophilic proteins, can more easily disrupt the surface-bound water barrier. Once these repulsive forces have been overcome, it is likely that other mussel foot proteins, rich in 3,4-dihydroxyphenylalanine (DOPA), can approach the surface and complete the adhesion process.

barrier — the Schottky barrier — formed at the junctions between a tungsten diselenide monolayer and the electric contacts, and found that the specific detectivity (a figure of merit used to quantify the response of photodetectors to light illumination) and the response speed of these devices strongly depend on the barrier height. They tuned the energy of the metal-semiconductor contacts by using two different metals and by testing the devices in vacuum and ambient conditions. Their results show that phototransistors having low Schottky barriers reach a detectivity that is higher than that of commercial photodetectors, but have a very slow response. On the other hand, a high barrier can improve the speed and the linearity of the device at the expense of a reduced sensitivity.

$L M$

\section{Artificial spin rotors}

Appl. Phys. Lett. 105, 042409 (2014)

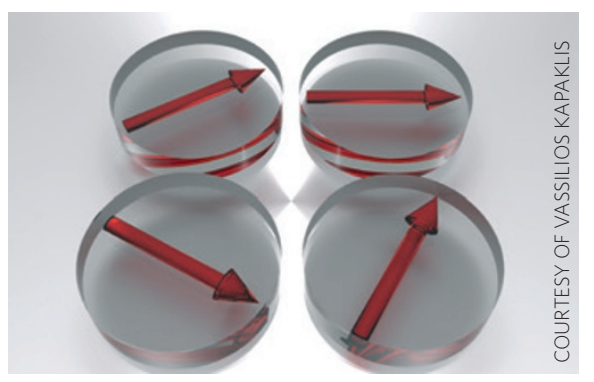

Lithographically fabricated arrays of nanoscale magnets have garnered attention as mesoscopic realizations of spin ice, a state of magnetic order that shares deep similarities with the thermodynamic properties of water ice. In such 'artificial' spin ices, nanoislands act as giant spins that can point in one of two directions ('up' or 'down'). Importantly, the islands interact with each other through the dipolar interaction, and thus mimic the frustrated behaviour of naturally occurring spin ice materials. The Ising symmetry of the spins - that is to say, the fact they can only point in one of two opposite directions - is crucial for ensuring this analogy holds. However, real magnets may also possess spins with a greater degree of freedom: for example, in some cases they may be free to rotate in the plane, and are referred to as $X Y$ spins. Now, Unnar Arnalds and colleagues fabricate an array of disc-shaped islands that behave as $X Y$-like superspins. These do not yet interact, but they do display thermal dynamics, by blocking and rotating freely at low and high temperature, respectively. More complicated artificial magnetic structures may therefore be just around the corner.

Written by Luigi Martiradonna, Olivia Nicoletti, Pep Pàmies, Alison Stoddart and Andrea Taroni. 